\title{
Nuevo equipo para medir la distribución de la densidad aparente de piezas cerámicas: DENSEXPLORER
}

\author{
${ }^{1}$ G. Mallol, ${ }^{1}$ J. Boix, ${ }^{1}$ D. Llorens, ${ }^{2}$ A. Poyatos y ${ }^{2}$ R. Bonaque \\ 'Instituto de Tecnología Cerámica UJI (Castellón) Campus Universitario Riu Sec, Avda. de Vicent Sos Baynat s/n, 12006 Castellón \\ ${ }^{2}$ MACER Cno. Estación s/n, 12550 Almazora
}

\begin{abstract}
Este trabajo ha participado en la 38 edición de los premios Alfa de Oro, otorgados por la Sociedad Española de Cerámica y Vidrio, durante la Feria Internacional de Cerámica de Valencia CEVISAMA 2014
\end{abstract}

Densexplorer es el primer dispositivo capaz de determinar la distribución de la densidad aparente de baldosas cerámicas industriales de gran tamaño, de forma no destructiva, no perjudicial para la salud, respetuosa con el medio ambiente, incrementando la productividad respecto a los equipos actuales. El sistema está basado en la medida puntual de la absorción de rayos $\mathrm{X}$ y del espesor de la pieza.

\section{Summary}

Densexplorer is the first device for industrial manufacturing plant capable of determining the distribution of bulk density of large ceramic tiles in a non-destructively way, which is not harmful to health, respectful with the environment and that increases the productivity of the process regarding to existing equipment. The system is based on the X-ray absorption technique, in which a material absorbs an amount of radiation depending on its thickness and density.

\section{INTRODUCCIÓN}

El comportamiento de las piezas cerámicas durante el proceso de fabricación, la mayor parte de las propiedades del producto final y gran parte de los defectos de fabricación más comunes dependen de la porosidad en crudo de las piezas.

Existe una relación directa entre porosidad, y la densidad aparente en crudo de las piezas. Por la mayor sencillez en la medida, se prefiere la de la densidad aparente a la de la porosidad para el control de la operación industrial de prensado. Este control, como todos los que se realizan en planta, deberá ser razonablemente económico, rápido, fiable, no perjudicial para la salud, respetuoso con el medio ambiente, y fácil de realizar e interpretar.

Densexplorer es una máquina que utiliza el método de absorción de rayos $X$ para la medida de densidad de las baldosas cerámicas. La elección de esta tecnología viene motivada por ser la más sencilla de implementar, más respetuosa con la salud y el medio ambiente que la que se viene utilizando habitualmente empleando mercurio, y por obtener medidas precisas y reproducibles.

El sistema se fundamenta en la ecuación de LambertBeer. Para una radiación monocromática, el cociente entre la intensidad de radiación que atraviesa la probeta y la intensidad de radiación incidente, depende del coeficiente de absorción del material, del espesor de la probeta y de su densidad aparente:

$$
\frac{I_{\lambda}}{I_{\lambda o}}=e^{-\mu \rho_{a p} e}
$$

donde:

- $\mathrm{I}_{\lambda}$ : Intensidad de radiación que atraviesa la probeta $(\mathrm{W} /$ $\left.\mathrm{m}^{2}\right)$

- $\mathrm{I}_{\lambda \circ}$ : Intensidad de radiación incidente $\left(\mathrm{W} / \mathrm{m}^{2}\right)$

- $\mu$ : Coeficiente de absorción de Rayos X $\left(\mathrm{m}^{2} / \mathrm{Kg}\right)$

- e: Espesor de la probeta (m)

- $\rho_{\mathrm{ap}}$ : Densidad aparente de la pieza $\left(\mathrm{Kg} / \mathrm{m}^{3}\right)$

El coeficiente de absorción $\mu$ depende de su composición química, para un determinado voltaje e intensidad de corriente eléctrica del tubo de rayos $\mathrm{X}$, $\mathrm{y}$ puede calcularse a partir del análisis químico del material y del conocimiento del coeficiente de absorción atómico de cada elemento. Además es necesario medir el espesor de la probeta con una precisión elevada y sin contacto, lo que puede conseguirse mediante el uso de telémetros láser adecuados.

En la Figura 1 en la página siguiente se ilustra esquemáticamente el sistema de medida desarrollado.

La pieza a medir se sitúa sobre una banda que la van transportando hacia la línea de medida de forma discontinua, paso a paso. El desplazamiento en cada paso depende de la calidad de la medida que se pretende alcanzar y del tiempo total de medida, variando entre 1 y10 $\mathrm{mm}$. A su vez, un tubo emisor de rayos $X$ se desplaza transversalmente en la pieza, escaneándola de izquierda a derecha y viceversa, a una velocidad máxima de $1000 \mathrm{~mm}$ por segundo. En el otro lado del tubo emisor se ubica un sensor de radiación, que mide 


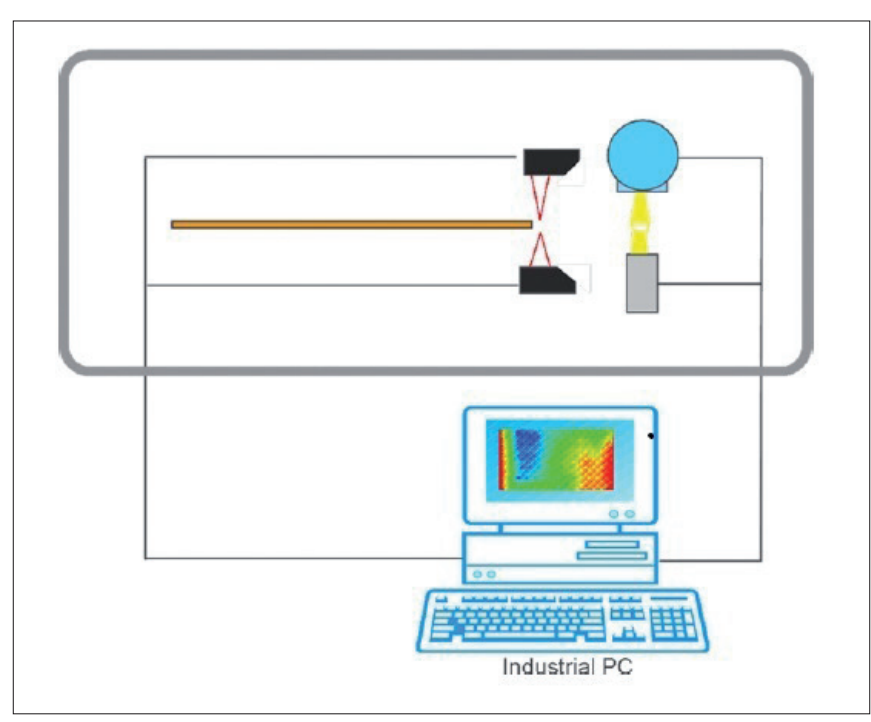

Figura 1. Esquema del dispositivo desarrollado

la cantidad de radiación que atraviesa la pieza. Un sistema de telemetría láser acompaña también al tubo realizando la medida de espesor.

A partir de la medida de la absorción de radiación y del espesor puntual en la pieza es posible obtener la distribución de la masa del polvo atomizado en la pieza, la distribución de la densidad aparente y la distribución del espesor de la pieza. Los datos son enviados a un PC, el cual trata los datos y los muestra al usuario, ofreciendo varias posibilidades de presentación .

El tubo de rayos $X$ tiene una potencia de $80 \mathrm{w}$. El equipo ha sido diseñado para cumplir con todos los requisitos de seguridad frente a radiación, y está certificada por el Consejo de Seguridad Nuclear para su uso industrial totalmente seguro, sin que el usuario requiera ningún tipo de formación reglada ni elemento de seguridad o medida (Figura 2).

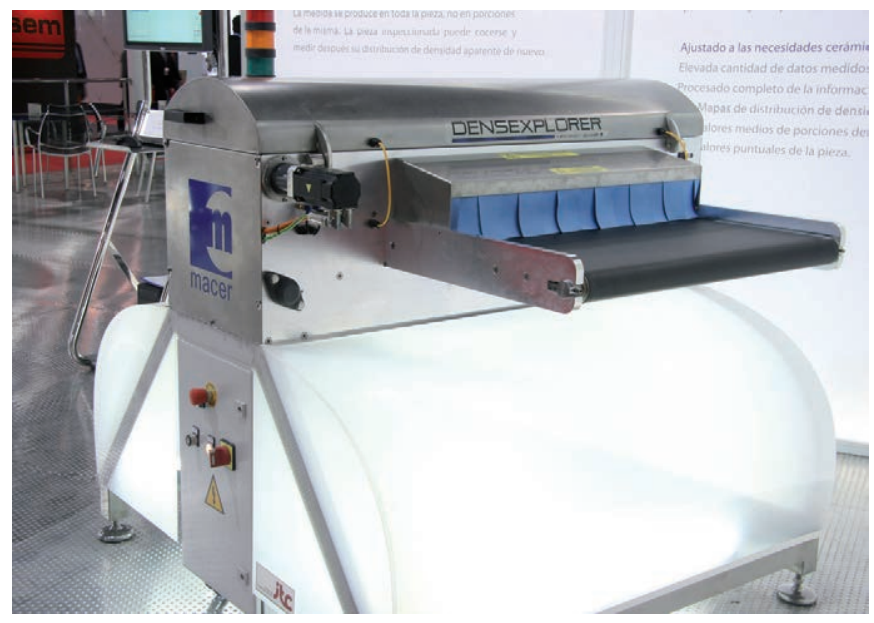

Figura 2. Equipo de medida construido.

\section{VIABILIDAD A ESCALA INDUSTRIAL}

Con vistas a evaluar la utilidad del instrumento para estudiar la operación de prensado de baldosas cerámicas, se analizaron varias piezas industriales con relieve y formato nominal $33 \mathrm{~cm} \times 33 \mathrm{~cm}$ obtenidas en diferentes condiciones de prensado. Para ello, se llevaron a cabo una serie de experimentos en una prensa industrial equipada con un molde penetrante convencional de cuatro salidas dotadas de punzones superiores iso-estáticos destinados a paliar las posibles deficiencias de la carga en los alvéolos. Los experimentos consistieron en la modificación de la presión máxima del ciclo de prensado y/o de la distribución de la carga de polvo atomizado en los alvéolos de la prensa.

\subsection{Medida de la distribución de la masa de polvo en la pieza}

En la Figura 3 se muestran la distribución de la masa de polvo en la pieza por unidad de superficie (producto $\rho_{\text {ap }}$ e) correspondientes a las cuatro piezas obtenidas en cada una de los experimentos s realizados, junto con los valores medios de la masa de polvo por unidad de superficie de cada pieza. Estas representaciones proporcionan información sobre la distribución de la carga de polvo atomizado en el seno de los alvéolos de la prensa en el momento de aplicar la presión máxima de prensado.

Como puede apreciarse, tanto la distribución de masa por unidad de superficie 1 , como los valores medios de este parámetro de cada pieza, en las dos primeras maniobras son muy similares, ya que la única diferencia entre ellas es la presión máxima de prensado alcanzada, habiéndose realizado la carga del polvo atomizado en las mismas condiciones. Cabe destacar que en ambas maniobras, la pieza correspondiente al plato 4 presenta una masa sensiblemente inferior a la del resto de piezas. Del mismo modo, se observa que todas las piezas, independientemente de la posición en la que han sido prensadas, presentan la carga ligeramente desplazada hacia la parte delantera del alvéolo.

El análisis de las distribuciones de masa correspondientes a las piezas de la maniobra 3 revela un aumento considerable de la masa introducida en los alvéolos y un desplazamiento de la carga hacia la parte trasera del molde, con respecto a los valores obtenidos en las maniobras 1 y 2 . Este cambio en la distribución de la masa se consiguió anulando la corrección en la posición de los punzones inferiores que se producía en las maniobras 1 y 2 al retroceder el sistema de carga de polvo. Dicha corrección, permitía eliminar una cierta cantidad del polvo depositado previamente, durante el avance del sistema de carga, en la parte trasera de los alvéolos.

Finalmente, la distribución de masas resultante de la maniobra 4 muestra que la carga de polvo atomizado se encontraba completamente desplazada hacia la parte delantera del molde, como consecuencia de una corrección excesiva de la posición de los punzones inferiores durante el retroceso del sistema de carga. Puede apreciarse que la masa media de polvo introducida en cada alvéolo, al contrario de lo que sucedía en la maniobra 3, disminuye considerablemente con especto a las maniobras 1 y 2 .

Hay que remarcar que en todas las maniobras realizadas, es siempre la pieza prensada en la posición 4 la que presenta una menor masa por unidad de superficie media. Del mismo modo, puede observarse que en el seno de las piezas prensadas en los alvéolos 1 y 4 existen zonas de baja masa por unidad de superficie que se mantienen, en mayor o menor medida, a lo largo de todas las maniobras realizadas (parte trasera derecha en las piezas del alvéolo 1 y parte trasera izquierda en las 


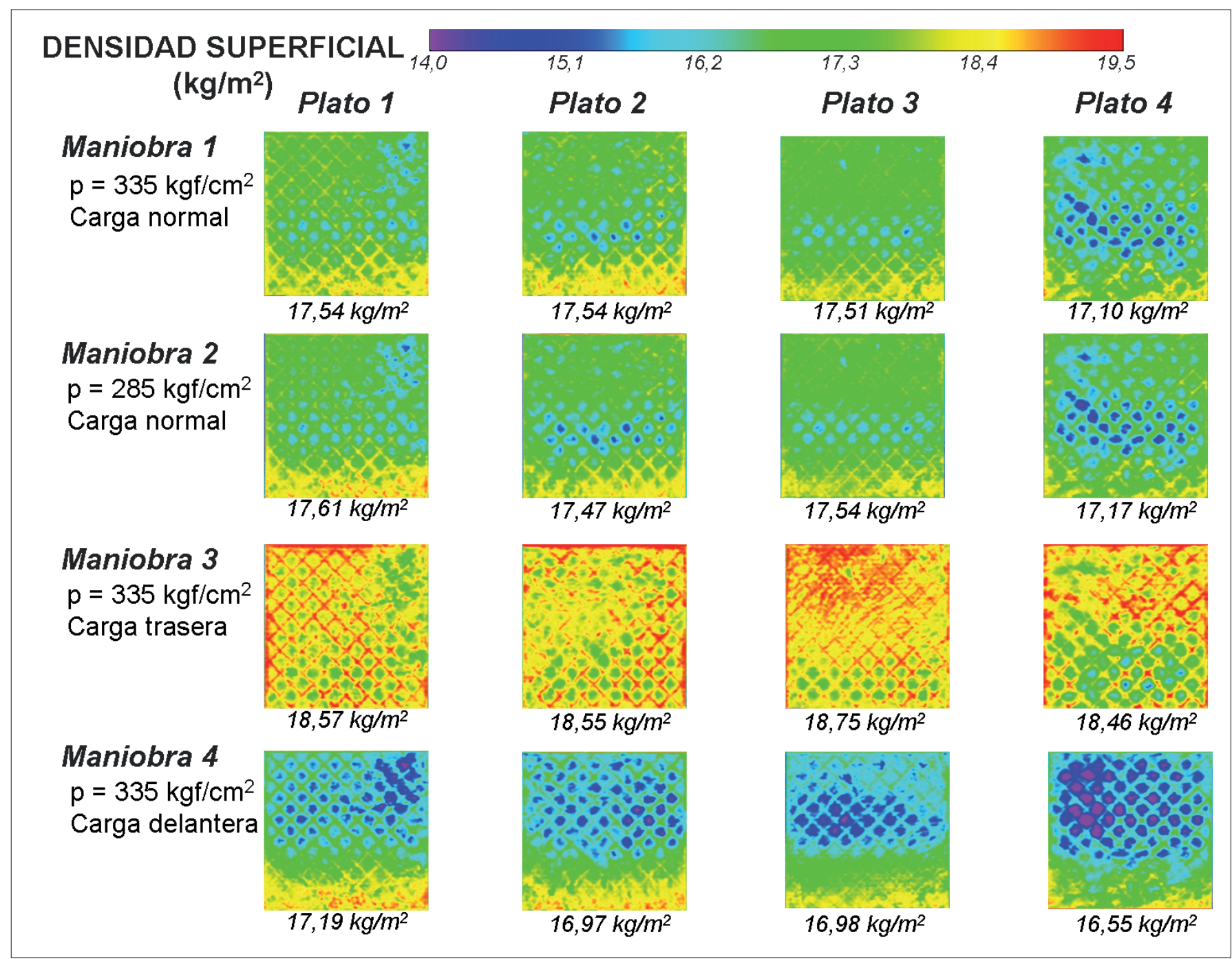

Figura 3. Distribución de la masa por unidad de superficie en el seno de las piezas obtenidas en diferentes condiciones de prensado.

piezas del alvéolo 4). Este hecho está asociado a la presencia de relieves en la cara vista de la baldosa y pone de manifiesto la importancia de controlar su diseño para asegurar una buena distribución de la carga.

\subsection{Medida de la distribución de espesores}

En la Figura 4 se representan las distribuciones de espesor obtenidas en las diferentes maniobras realizadas. Si se comparan estos mapas de espesor con las distribuciones de masa mostradas en la Figura 3 puede comprobarse que ambas variables están íntimamente relacionadas. Las zonas de las piezas con mayor cantidad de material son las que al finalizar el ciclo de prensado presentan un mayor espesor. Al analizar las distribuciones de espesor resultantes de las maniobras 1 y 2, puede observarse que éstas tan sólo se diferencian en los valores medios del espesor alcanzado. Las piezas de la maniobra 1 poseen espesores ligeramente inferiores a las de la maniobra 2 (la diferencia media es de $0,08 \mathrm{~mm}$ ) debido a la menor presión máxima de prensado ejercida sobre las mismas. En efecto, para una misma cantidad de polvo atomizado existente en los alvéolos (ver Figura 3) y una velocidad de desplazamiento de la traviesa de la prensa constante, es el ciclo de prensado de la maniobra 2 el que alcanza antes los valores de presión de consigna y, por lo tanto, proporciona piezas de espesor ligeramente superior. En cuanto a las piezas obtenidas en la maniobra 3, éstas presentan un espesor medio sensiblemente inferior al de las obtenidas en las maniobras 1 y 2, como consecuencia de la menor cantidad de material existente en los alvéolos al finalizar el ciclo de carga. Por el contrario, los menores espesores de las piezas de la maniobra 4 están asociados a la menor cantidad de polvo cargado en los alvéolos.

Al igual que sucede con la distribución de carga, es importante destacar que, independientemente de la presión máxima del ciclo de prensado, las distribuciones de espesor de las maniobras 1 y 2 son prácticamente idénticas, lo cual pone de manifiesto la gran robustez de los sistemas de carga de polvo atomizado utilizados hoy en día en las prensas industriales.

Es decir, aunque pueden existir deficiencias en la distribución de la carga en los alvéolos de la prensa, debido a una incorrecta regulación del sistema de carga, dichas diferencias de carga no son aleatorias, manteniéndose constantes a lo largo del tiempo. Este comportamiento tan robusto asegura que las modificaciones realizadas en el proceso de carga de polvo atomizado queden inmediatamente reflejadas en los soportes recién prensados y que se mantengan 


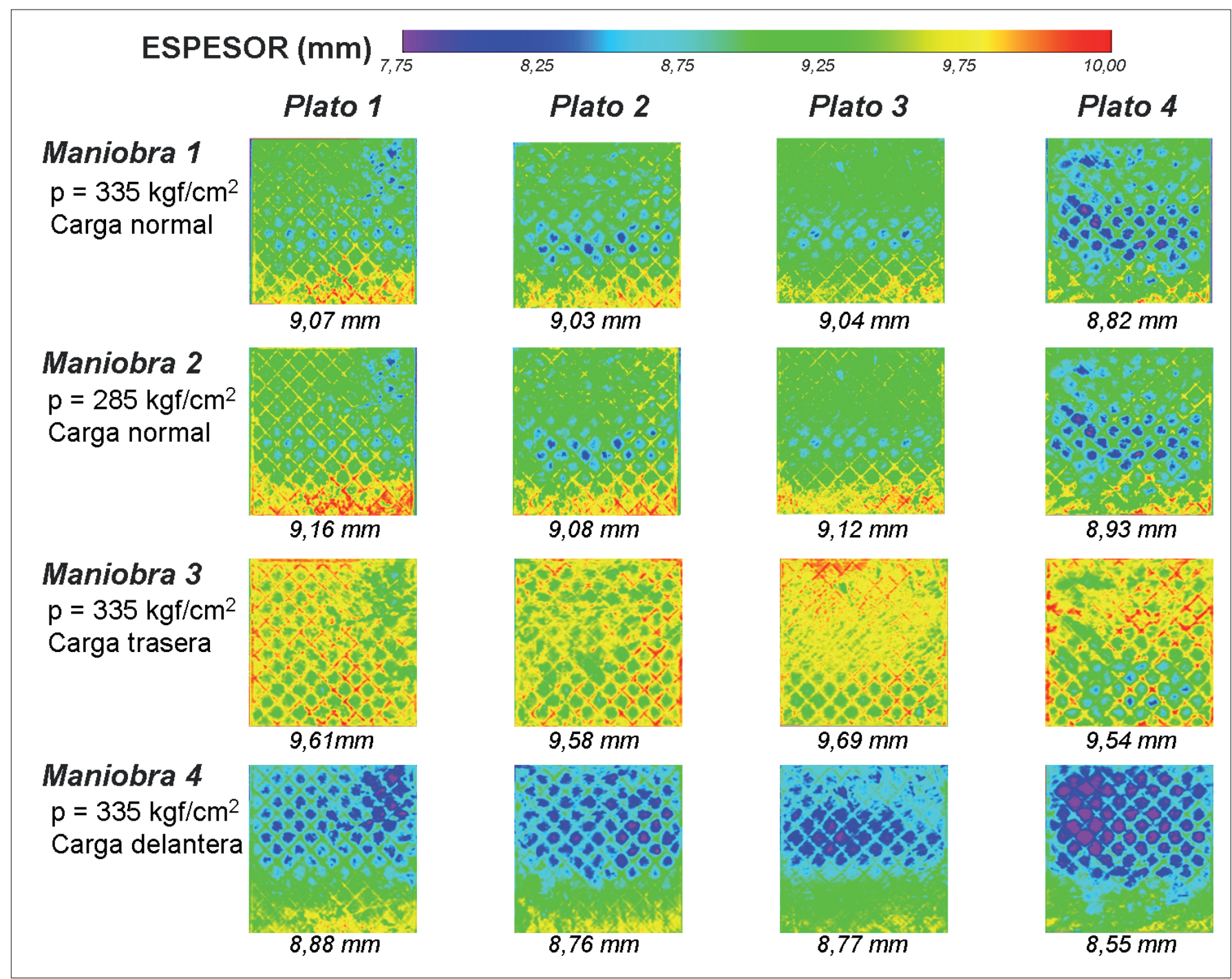

Figura 4. Distribución de espesores en el seno de las piezas obtenidas en diferentes condiciones de prensado.

a lo largo de la producción, siempre y cuando no se modifique la naturaleza del polvo de prensas.

Ahora bien, debido a su carácter destructivo y manual, los métodos empleados en el control de la operación de prensado a nivel industrial no permiten inspeccionar con suficiente detalle los soportes recién prensados y, por esta razón, los defectos de fabricación asociados a una incorrecta distribución de polvo en los alvéolos de la prensa siguen siendo comunes hoy en día. En este sentido, el método de absorción de rayos $\mathrm{X}$, en la medida en que permite obtener mapas completos de la distribución de tres variables esenciales para el control de la operación de prensado (masa, espesor y densidad aparente), se convierte en una herramienta de gran utilidad para abordar los problemas de fabricación asociados a un incorrecto desarrollo de la etapa de prensado.

\subsection{Medida de la distribución de la densidad aparente}

Finalmente en la Figura 5 se recogen los mapas de distribución de la densidad aparente de las piezas estudiadas. Como puede verse, la densidad aparente media de las piezas obtenidas depende exclusivamente de la presión máxima de prensado, en cambio, la distribución de densidades está directamente relacionada con la distribución del polvo en el interior del alvéolo. Por un lado, la densidad aparente de las piezas obtenidas en la maniobra 1 es muy similar a la obtenida en las maniobras 3 y 4, puesto que la presión máxima aplicada fue en los tres casos de $335 \mathrm{~kg} / \mathrm{cm}^{2}$. Sin embargo, las piezas correspondientes a la maniobra 2 presentan una densidad aparente media inferior, al haber estado prensadas a $285 \mathrm{~kg} / \mathrm{cm}^{2}$. Y por otro lado, puede apreciarse que, al desplazar la carga dentro del alvéolo (maniobras 3 y 4), la distribución de densidad aparente se ve modificada en el mismo sentido.

Los resultados derivados de estas maniobras, ponen de manifiesto que el análisis detallado de las distribuciones de densidad aparente por absorción de rayos $\mathrm{X}$ permitiría evaluar si una determinada pieza es susceptible de presentar defectos de fabricación asociados a una incorrecta distribución del polvo en el alvéolo de la prensa.

\subsection{Identificación de defectos de prensado y cocción en baldosas cerámicas de gran formato}

En la Figura 6 se muestran las distribuciones de espesor y densidad aparente de dos piezas lisas de gres porcelánico de formato nominal $45 \mathrm{~cm}$ x $67 \mathrm{~cm}$ obtenidas en una prensa industrial equipada con un molde de doble matriz con 


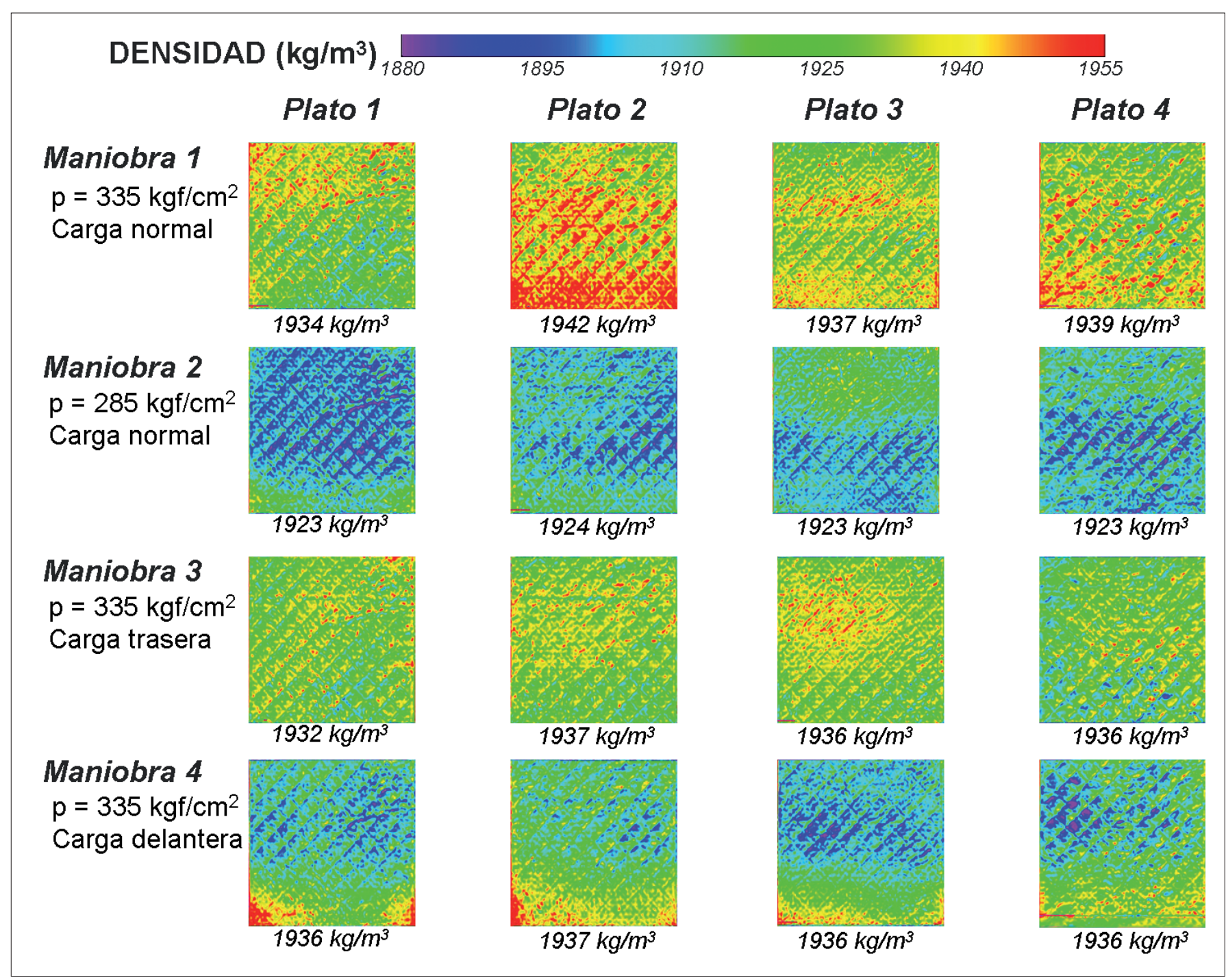

Figura 5. Distribución de densidades aparentes en el seno de las piezas obtenidas en diferentes condiciones de prensado.

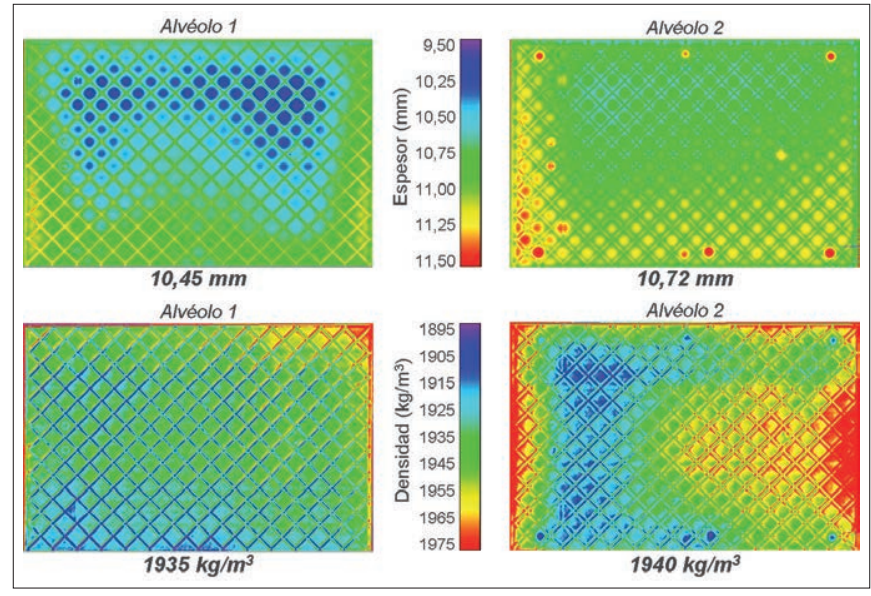

Figura 6. Distribuciones de espesor (arriba) y de densidad aparente en seco (abajo) de dos piezas de gres porcelánico crudas.

punzones inferiores iso-estáticos. La pieza prensada en el alvéolo 1 presentaba una distribución homogénea de la densidad aparente, en cambio, la pieza procesada en el alvéolo 2 , como consecuencia de un funcionamiento inadecuado del punzón iso-estático, mostraba una distribución heterogénea de la densidad aparente, la cual originaba defectos de descuadre en el producto final.

La interpretación del defecto puede completarse con la información aportada por la distribución de espesores de ambas piezas. Como puede comprobarse, la pieza 1 presentaba unas diferencias de espesor muy marcadas, asociadas a la compensación de presiones ejercida por el punzón iso-estático sobre el lecho de polvo. El efecto del punzón iso-estático originaba una distribución de densidades con unas diferencias medias máximas dentro de la pieza inferiores a $20 \mathrm{~kg} / \mathrm{m}^{3}$. Sin embargo, durante el prensado de la pieza 2, no se produjo dicha compensación, dando como resultado una pieza con una densidad aparente media muy similar a la de la obtenida en el alvéolo 1, pero con un espesor medio superior y una distribución de densidad aparente con diferencias medias máximas entorno a $50 \mathrm{~kg} / \mathrm{m}^{3}$.

Sin embargo, durante el prensado de la pieza 2, no se produjo dicha compensación (la forma redondeada de la distribución de espesores las zonas situadas entre las costillas denota una falta de aceite en el punzón iso-estático que permitió la deformación del revestimiento de goma que lo recubre), dando como resultado una pieza con una densidad aparente media muy similar a la de la obtenida en el alvéolo 1 , pero con un espesor medio superior y una distribución de 


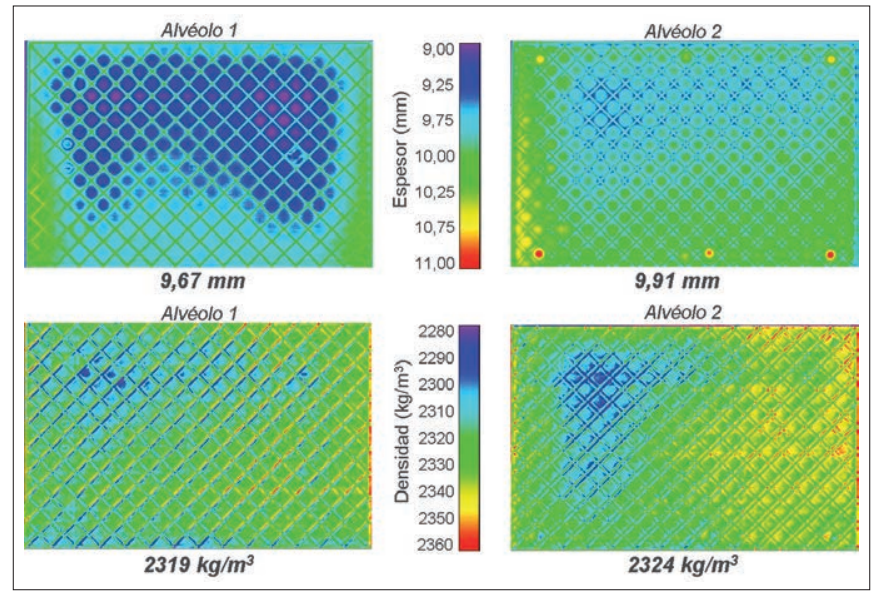

Figura 7. Distribuciones de espesor (arriba) y de densidad aparente (abajo) de dos piezas de gres porcelánico cocidas.

densidad aparente con diferencias medias máximas entorno a $50 \mathrm{~kg} / \mathrm{m}^{3}$.

El carácter no destructivo del método permitió analizar las mismas piezas tras someterlas a un ciclo de cocción industrial con una temperatura máxima de $1200^{\circ} \mathrm{C}$ y evaluar, de este modo, la influencia del proceso de sinterización sobre las propiedades del producto final. En la Figura 7 se representan las distribuciones de espesor y de densidad aparente en cocido de las dos piezas estudiadas. La distribución de densidades aparentes en cocido muestra que las heterogeneidades generadas durante el prensado en la pieza 2 seguían presentes tras la cocción, aunque en una menor magnitud, como consecuencia de la baja influencia de la densidad aparente en seco sobre la densidad aparente en cocido que presentan las composiciones de gres porcelánico a elevadas temperaturas de cocción.

El ejemplo que se acaba de presentar muestra la utilidad del método de absorción de rayos $\mathrm{X}$ para identificar, entre otros, el origen de los defectos de fabricación asociados a una falta de estabilidad dimensional del producto final. En efecto, la posibilidad de caracterizar las mismas piezas, antes y después de la cocción, permite discernir si un determinado defecto es debido a un incorrecto desarrollo de la operación de prensado o, por el contrario, aparece como consecuencia de una mala regulación del horno.

\section{CONCLUSIONES}

Se ha desarrollado una máquina capaz de medir la distribución de densidad aparente en una baldosa cerámica, empleando la tecnología de absorción de rayos $\mathrm{X}$, cuyas principales características son:

- Reduce considerablemente el tiempo de medición respecto a los sistemas actuales (entre un $60-80 \%$ ), lo que agiliza el tiempo de respuesta en el sistema productivo y por tanto incremente su productividad.

- Permite obtener mapas de distribución de densidad aparente de toda la pieza, mediante medida no destructiva en baldosas cerámicas.

- Proporciona información adicional de espesor y distribución de masa por unidad de superficie, lo que es de gran interés para la optimización del proceso de conformado de baldosas cerámicas.

- Detecta defectos puntuales relacionados con una distribución de densidad aparente heterogénea causada por una operación de prensado inapropiada.

- El carácter no destructivo del sistema permite examinar piezas cocidas que han sido previamente examinadas en crudo , para así evaluar el efecto de las etapas de cocción y prensado en las propiedades del producto final.

- Es respetuosa con la salud del trabajador y el medio ambiente. 